\title{
Seeing into your Teams: An Instructor Interface to Support Team Learning
}

\author{
Patricia Kristine Sheridan ${ }^{1,2}$, Aidan Malone ${ }^{1,3}$, Doug Reeve ${ }^{1,2}$, and Greg Evans ${ }^{1,2}$ \\ ${ }^{1}$ Institute for Leadership Education in Engineering, ${ }^{2}$ Dept. of Chemical Engineering and Applied Chemistry, \\ ${ }^{3}$ Division of Engineering Science \\ patricia.sheridan@utoronto.ca
}

\begin{abstract}
This paper outlines the design of a new instructor interface that has been added to the on-line Team-effectiveness Learning System (TELS) at the University of Toronto. TELS is a tool that supports teambased project courses by facilitating the development of individual team-effectiveness competencies in students within their teams. Instructors saw the system as beneficial for student growth, but they also saw opportunities for them to get a better understanding of the state of their student teams. As a result, TELS has now developed an instructor interface to let instructors "see" into their teams. The instructor interface has been adopted by four courses since its development.
\end{abstract}

Keywords: team-effectiveness, team-based projects, supporting student teams.

\section{INTRODUCTION}

This paper presents the design of a new instructor interface to assist instructors of team-based projects in better supporting their student teams. This interface was designed in response to the comments of design teaching assistants and instructors at the University of Toronto. Instructors could generally identify which teams were doing well and which were doing poorly, but they were unable to analytically determine why the teams were functioning the way they were based on specific behaviours of team-effectiveness in their limited contact with them [1]. As a result, for student teams that were heading towards dysfunction, or teams that were performing functionally but sub-optimally these instructors were ill-prepared to support these teams.

Interfaces to provide instructors information as to how their student teams are functioning exist within other online self- and peer-assessment systems. Due to the differences in the focus of the assessments used in these systems, we desired to create an interface for instructors that matched the founding principles of our assessment focus. As a result, our interface could not focus primarily on assessing student quantity of contribution to the project, but needed to assess the student contribution to creating an effective team-environment. Thus, we chose to respond to the instructors request for more information about their teams from the perspective of team cohesion, rather than individual contributions to deliverables.

\section{TEAM-EFFECTIVENESS LEARNING SYSTEM}

The Team-effectiveness Learning System (TELS) is an on-line tool that supports student learning and development of effective team-member behaviour within project teams by allowing students to provide feedback to their team mates on their behaviour and performance [2]. The TELS facilitates within-team, formative self- and peer-assessments using a 3 -aspect, 12 -item behavioural inventory of team-member effectiveness, presented in [2]. Students who use the TELS complete the 12-item inventory for all students in their project team as well as provide some holistic feedback to them on their performance over the course of the project. The system anonymises the feedback, and provides students with a comparative representation of their received self- and peer-assessments along the 12-item inventory as well as the textual holistic feedback provided by their peers. Based on this feedback, the system identifies the 3 behavioural competencies that the student needs to improve most immediately and connects them directly to online resources that correspond to these competencies.

\subsection{System Use}

The TELS is currently in use in four courses at the University of Toronto, and has been tested extensively in the first year design courses for the past 3 years. The system was designed particularly for these design courses as they have class sizes of 250-1000 students, making it difficult for an instructor to facilitate individual team feedback and development sessions with each team, or each student, within the demands of course resources.

The TELS inventory is conducted twice during each course - first at the mid-point of the course, and second after submission of the final deliverable. The objective in spacing these assessments this way is to allow students (over a 13-week semester) to have spent sufficient time working with their team to be able to provide quality feedback in their first assessment, as well as to have 
enough time to act upon their feedback and demonstrate improved performance before being assessed for a second time.

Students have access to the feedback from both assessments at the end of the course and are able to see the change in their perceived performance based on any specific efforts they have pursued.

\section{INSTRUCTOR INTERFACE}

An instructor interface was designed to provide instructors and teaching assistants (TAs) supporting students in their team-based projects, with insight into the functioning of their teams. Previous studies on teaching assistants abilities to assess team-member effectiveness have shown that teaching assistants have limited ability to assess individual student's functioning in teams [1]; they are able to determine if a team is doing very well, or heading towards crisis, but are not able to readily determine how everyone in the team is creating this situation. Part of this is due to juggling this additional assessment load on top of the already existing cognitive load of supporting student learning of the technical and/or communication material of their projects. Part of this is due to the fact that the majority of teamwork occurs outside of tutorial work periods and outside of instructor/TA observation time. As a result, the ability to provide instructors with information as to the effectiveness of the teams they support from a student perspective would greatly increase their ability to appropriately and adequately guide their students towards creating effective work environments.

The instructor interface uses the students' feedback provided within the TELS inventory assessments to compose a series of diagnostic visuals that demonstrate the relationships between, and competencies of, the team members in each project team. These visuals aim to provide an instructor the ability to 'see': 1) how closely the students have bonded as team members, 2) any differences in self- and peer-assessments that might signify a contribution or motivation issue on the part of a team member, and 3) an appropriate starting person or topic to begin conversing with dysfunctional teams about how to improve their team-effectiveness.

Please note that all visuals and examples contained within this paper are of hypothetical students.

\subsection{Team Cohesion Diagram}

The team cohesion diagram is meant to give the instructor a quick overview of the team's situation. It responds to the question: Is the team a cohesive unit or are there cliques, or divisions between different members?

CEEA15; Paper 055

McMaster University; May 31 - June 3, 2015- 2 of 4 -
The team cohesion diagram is a type of sociogram that looks at the interconnectedness of the team members. An example of this diagram for a dysfunctional team can be seen in Figure 1. Each of the five team members is connected to each other by a link. The thickness and length of each link are calculated based on Alba's clique detection methodology [3], and are meant to demonstrate the strength of the relationship between the pairs of team members. The strength of the relationship is determined by the amount of agreement between the self- and peer assessments. Two students who see each other the same way as they see themselves are assumed to have a stronger relationship with each other; this stronger relationship allows for a greater understanding of each other that is reflected in the agreement between their self- and peerassesments.

Students who have greater agreement between their self- and peer-assessments will have thicker shorter (strong) links between team members and students who have less agreement will have longer thinner (tenuous) links. You can imagine the team like a molecule, students that understand each other have stronger bonds between them, and students that don't have weaker bonds.

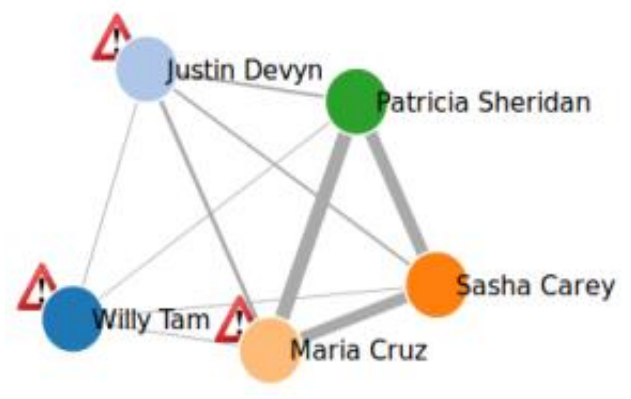

Figure 1: Diagram of a less effective team of five, where three team members have formed a clique within the team, and two team members are not effectively connected with the other team members.

In the example shown in Figure 1, you can see that three of the five students have a good understanding of each other \{Patricia Sheridan, Sasha Carey, and Maria Cruz\}; in many ways these three team members have formed a clique within their team of five. Both Justin Devyn and Willy Tam have thinner longer links between them and their team members showing that there is a lack of agreement between their perception of their effectiveness in the team and their team members.

Were this example team an effective team, where there was agreement between individual students' self- and peer-assessments, the team cohesion diagram would look like a pentagon with equally thick and short lines connecting every member to each other. From these diagram configurations, an instructor can quickly visualize 
whether the team is "together". If any one student is not closely bonded with the others, it is visible on inspection.

The team cohesion diagram also flags students that have substantive differences in their self- and peerassessments. These are shown as alert signs beside the students name and node on the diagram. In Figure 1, Willy Tam, Justin Devyn, and Maria Cruz have all been flagged as warranting further inspection. These students are flagged as potential intervention points for an instructor in the case of a near-dysfunctional team. These would be good students to have a conversation with to understand the team dynamic and determine how to best support them and their team in becoming more effective.

\subsection{Comparative Student Assessments}

To better understand the differences in student perception that lead to the lack of agreement seen in the team cohesion diagram, instructors are also provided with comparative students assessments. The objective of these per-student charts is to show an instructor the difference between the self- and peer-assessments provided for a student on a competency aspect level.

For each aspect two bars are provided. The top bar is the student's average self-assessment across the aspect ( $n=4$ competencies) and is marked as the self-assessment by having one head to the left of the bar. The bottom bars are the student's average peer-assessments across the aspect $(n=4$ competencies $x$ number of peer team members). It is marked with three heads to the left of the bar to show that it is a peer assessment.

These self- and peer-assessment differences are used to flag students who are not being seen to be working effectively in the team. There are three types of students we aim to identify: the over assessor, the under assessor, and the student with inconsistent assessments. Each example discussed below is of a student that was flagged by the system as having a discrepancy in their ratings; please note the alert signs beside each students' circle on the steam cohesion diagram in Figure 1.

An example of a student over-assessor in the team shown in Figure 1, is Willy Tam. As can be seen in Figure 2, Willy has consistently rated himself as perfect (4/4) across all three aspects. By contrast his peers have rated his behaviour within the team very poorly (approx. 1/4). Willy Tam in this case either thinks he is perfect and is oblivious to his faults, or more likely, is an example of a student who is not engaged with the team. As a result, when asked to complete the assessments, Willy provided thoughtless ratings of perfect for himself while the others on the team reflected on his limited engagement.

An example of a student under-assessor in the team shown in Figure 1, is Maria Cruz. As can be seen in Figure 3, Maria has consistently rated herself as not performing as well as her peers perceive her. Students who continually self-assess lower than their peers are usually not students that an instructor needs to be immediately concerned about, as they are likely working twice as hard on their team work to match their personal perception of an effective team member. However, students like this can be of concern if their continually poor self-perception leads to issues of lack of motivation, and not feeling they are good enough for the team which may lead to them "checking out" from the team.

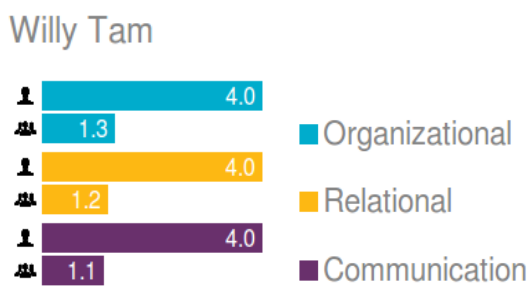

Figure 2: An example of an over-assessor student who self-assesses high, and is peer-assessed very low. The colour legend for the aspects shown in this figure also applies to Figures 3, 4, and 5.

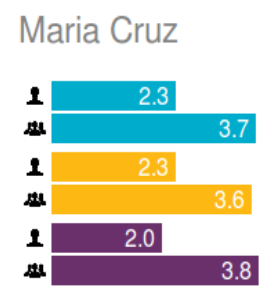

Figure 3: An example of an under-assessor student who self-assesses low, and is peer-assessed high.

\section{Justin Devyn}

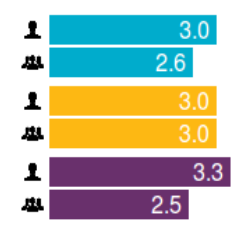

Figure 4: An example of a student who had inconsistent assessments provided by their peers. As can be seen in this figure there are no visually identifiable markers for a student with these kind of ratings other than the alerts on the team cohesion diagram, Figure 1.

An example of a student with inconsistent assessments from the team shown in Figure 1, is Justin Devyn. He is a student where the self- and peer-assessments show no visually identifiable discrepancy. Inconsistent assessments (where a student is perceived as highly effective by one team-member and not effective by another) can easily hide when averaged across an aspect. As a result, the system watches for cases like this that will not be readily visible to an instructor. A student who flags as having 


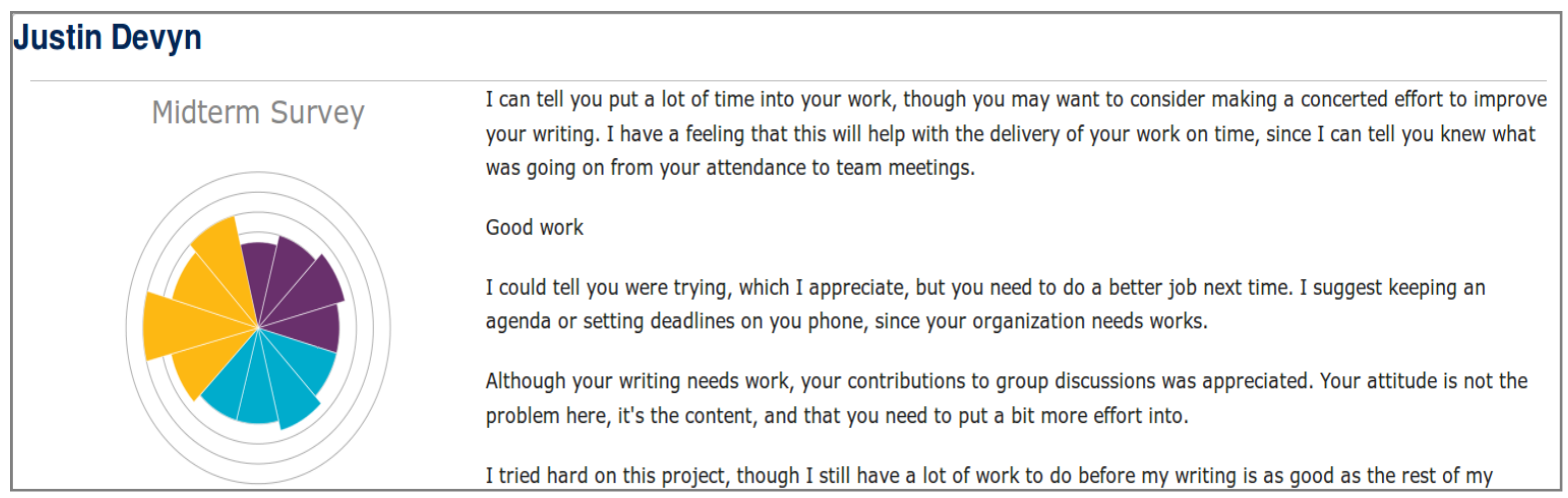

Figure 5:Student Peer-Feedback display for instructors with a competency-based radar plot on the left and student holistic feedback provided on the right.

inconsistent assessments may be part of a clique like the one shown in Figure 1, or may simply have drastically different relationships with their team members. As a result, they may work effectively with those they get along with but not with others. While these kinds of assessments are not visible on inspection of the chart, they are detected and flagged by the system for an instructor.

\subsection{Student Peer Feedback}

Once an instructor can see that there are issues relating to team-effectiveness within a team, they can delve into each student's peer-assessments to get more information about the situation. From the previous two visuals an instructor can see how togethered the team members are, and see if there are any strong differences in students' perceptions of their and their peers performance. The information provided in this visual gives an idea of who an instructor might want to start a conversation with to determine how to best support the team in becoming more effective. The student peer feedback aims to provide an instructor with the topics they might want to discuss with the students flagged previously, as in less effective situations students tend to provide specific examples of what is not working in the textual feedback.

Looking at the student peer feedback for each individual, Figure 5, an instructor is provided with: a radar plot of the student's performance along each of the 12 competencies of the inventory (left-hand side), and the verbatim textual feedback provided to the student by their peers (right-hand side). If the instructor mouses-over any of the behaviours in the radar plot, the name of the behaviour and the average peer assessment for that behavior will display. Therefore, an instructor can determine exactly which behaviours the student is poor at demonstrating, and compare these to the textual examples provided to gain a more complete picture of how the student is or is not effective at contributing to the team environment.

CEEA15; Paper 055

McMaster University; May 31 - June 3, 2015- 4 of 4 -

\section{USE OF THE INSTRUCTOR INTERFACE}

Launched in September 2014, the instructor interface is currently being used in 4 courses, with approximately 50 course instructors and teaching assistants. Perceptions and utility of the instructor interface are currently being investigated. Introduction of the interface into these courses was met very positively.

\section{CONCLUSION}

An instructor interface has been designed and incorporated into the Team-effectiveness Learning System to provide instructors with more information about how their teams are functioning. The interface flags potential sources of team dysfunction based on student self- and peer-assessments and provides instructors with recommended starting points for conversations with students about their team-effectiveness.

\section{Acknowledgements}

This research supported in part by the Ontario Graduate Scholarship and the University of Toronto Engineering Instructional Innovation Program.

\section{References}

[1] P. K. Sheridan, D. Reeve and G. Evans, "Understanding Teaching Assistants' Assessment of Individual Teamwork Performance," in American Society for Engineering Education Annual Conference and Exhibition, Indianapolis, IN, 2014.

[2] P. K. Sheridan, P. M. To, G. J. Evans and D. W. Reeve, "An On-line Team-effectiveness Learning System," in Canadian Engineering Education Association Annual Conference, Canmore, AB, 2014.

[3] R. D. Alba, "A graph-theoretic definition of a sociometric clique," The Journal of Mathematical Sociology, vol. 3, no. 1, pp. 113-126, 1973. 